\title{
The influence of healthcare provider's autonomy support, autonomous motivation and competence on self-care behaviors in kidney transplant patients based on self-determination theory
}

\author{
Sunyoung Son ${ }^{1}$, Man ki Juㅜ, Mi Kyung Sim² \\ ${ }^{1}$ Division of Transplantation, Department of Surgery, Gangnam Severance Hospital, Seoul, Korea \\ ${ }^{2}$ Department of Nursing, Shinsung University, Dangjin, Korea
}

Background: It is important for transplant patients to perform continuous self-care behavior after transplantation. The self-determination theory is that explains the persistence factors of health behavior of chronically ill patients. This study was conducted to identify the factors of the self-determination theory of kidney transplant patients and to prepare basic data to improve self-care behavior of them.

Methods: The subjects of this study were 79 transplant patients who underwent follow-up care in an outpatient clinic after receiving a kidney transplant at one $\mathrm{G}$ university hospital in Seoul. Survey research was done using the "Health care climate questionnaire," "autonomous motivation," "Perceived competence scale" tools, and "self-management" tools. The data collected from April 20, 2020 to August 20, and the collected data were analyzed using SPSS ver. 21.0 for windows.

Results: The average age of the subjects was 54.1 years (27-78 years), and male patients were 46 (58.2\%). The duration after transplantation was 5.25 years. The factors a significant correlation was confirmed were Healthcare provider's autonomy support and autonomous motivation $(r=0.39, P<0.001)$, healthcare provider's autonomy and competence $(r=0.29, P<0.05)$, healthcare) , Healthcare provider's autonomy and self-care behavior $(r=0.34, p<0.01)$, autonomous motivation and competence $(r=0.39$, $P<0.001)$, autonomous motivation and self-care behavior $(r=0.47, P<0.001)$, competence and self-care behavior $(r=0.44, P<0.001)$. As a result of multiple regression analysis, the factors that have the greatest influence on self-care behavior were identified in the order of competence $(\beta=0.377, \mathrm{P}<0.01)$, and autonomous motivation $(\beta=0.293, \mathrm{P}<0.01)$. The total explanatory power was $30.1 \%$. Conclusions: As a result of this study, it was consistent with the results of previous studies that autonomous motivation increased competence and continuation self-care behavior. This could contribute to the development of an intervention program for improving the self-care behavior of patients.

Corresponding author: Sunyoung Son

E-mail: otc@yuhs.ac 